\title{
Peripheral Hearing Loss and Its Association with Cognition among Ethnic Chinese Older Adults
}

\author{
Sean Olivia Nicholas ${ }^{a}$ Emily Jiali Koh ${ }^{a}$ Shiou Liang Wee ${ }^{a, b, c}$ \\ Robert H. Eikelboom ${ }^{\text {d, e, f }}$ Dona M. P. Jayakody ${ }^{d, e}$ Frank Ling, ${ }^{g, h}$ \\ Tze Pin Nga, ${ }^{a}$ Rebecca L. Heywood ${ }^{d, e, j, k}$
}

${ }^{a}$ Geriatric Education and Research Institute (GERI), Singapore, Singapore; bSingapore Institute of Technology, Health and Social Sciences Cluster, Singapore, Singapore; ' ${ }^{\mathrm{D}}$ uke-NUS Medical School, Singapore, Singapore;

dEar Science Institute Australia, Subiaco, WA, Australia; 'Ear Sciences Centre, Medical School, The University of Western Australia, Subiaco, WA, Australia; ${ }^{\text {DDepartment }}$ of Speech Language Pathology and Audiology, University of Pretoria, Pretoria, South Africa; ${ }^{9}$ Cochlear Center for Hearing and Public Health, Johns Hopkins Bloomberg School of Public Health, Baltimore, MD, USA; ' Departments of Otolaryngology, Medicine, Mental Health, and Epidemiology, Johns Hopkins University, Baltimore, MD, USA; 'Department of Psychological Medicine, Gerontology Research Program, National University of Singapore (NUS), Singapore, Singapore; 'Department of Otolaryngology, Ng Teng Fong General Hospital, Singapore, Singapore; 'Yong Loo Lin School of Medicine, National University of Singapore, Singapore, Singapore

\section{Keywords}

Hearing loss · Cognition · Older adults

\begin{abstract}
Introduction: Many studies on hearing loss $(\mathrm{HL})$ and cognition are limited by subjective hearing assessments and verbally administered cognition tests, the majority of the document findings in Western populations. This study aimed to assess the association of $\mathrm{HL}$ with cognitive impairment among ethnic Chinese Singaporean older adults using visually presented cognitive tests. Methods: The hearing of community-dwelling older adults was assessed using pure tone audiometry. Cognitive function was assessed using the Computerized Cambridge Cognitive Test Battery (CANTAB). Multiple regression analyses examined the association between hearing and cognitive function, adjusted for age, edu-
\end{abstract}

cation, and gender. Results: HL (pure-tone average [PTA] of thresholds at $0.5,1,2$, and $4 \mathrm{kHz}$ in the better ear, BE4PTA) was associated with reduced performance in delayed matching and multitasking tasks $(\beta=-0.25, p=0.019$, and $\beta=0.02$, $p=0.023$, respectively). Moderate to severe $\mathrm{HL}$ was associated with reduced performance in delayed matching and verbal recall memory tasks $(\beta=-10.6, p=0.019$, and $\beta=$ $-0.28, p=0.042)$. High-frequency $\mathrm{HL}$ was associated with reduced performance in the spatial working memory task ( $\beta=$ $0.004, p=0.022$ ). All-frequency $\mathrm{HL}$ was associated with reduced performance in spatial working memory and multitasking ( $\beta=0.01, p=0.040$, and $\beta=0.02, p=0.048$ ). Conclusion: Similar to Western populations, HL among tonal language-speaking ethnic Chinese was associated with worse performance in tasks requiring working memory and executive function.

(c) 2021 The Author(s)

Published by S. Karger AG, Basel karger@karger.com www.karger.com/dem

Karger $\stackrel{\text { ' }}{5}$

BOPEN ACCESS
(C) 2021 The Author(s)

Published by S. Karger AG, Basel

This is an Open Access article licensed under the Creative Commons Attribution-NonCommercial-4.0 International License (CC BY-NC) (http://www.karger.com/Services/OpenAccessLicense), applicable to the online version of the article only. Usage and distribution for commercial purposes requires written permission. 


\section{Introduction}

A growing number of cross-sectional, longitudinal, and meta-analytical studies have demonstrated a relationship between peripheral HL and cognitive impairment among older adults [1-5]. Furthermore, HL has been associated with risk of incident dementia and Alzheimer's disease $[6,7]$. The mechanism for this remains poorly understood $[8,9]$.

Much of the relevant research has methodological drawbacks in the testing of HL and cognition [8]. Selfreported HL and subjective hearing tests may not accurately characterize hearing status. Verbally administered cognitive tests could overestimate the strength of the association [10-12]. Researchers have attempted to overcome this with test batteries that are visually presented or have been adapted for use in hearing-impaired subjects $[9,13,14]$. Computerized test batteries have also been employed $[15,16]$.

The literature pertaining to Asian populations is sparse and documents conflicting results $[17,18]$. The association of HL with cognition could vary in different populations depending on the mechanisms involved. Proposed pathways include impoverished sound input resulting in increased cognitive load, sensory decline causing increased brain atrophy, and social isolation [19]. Genetics and sociocultural contexts such as stigma associated with $\mathrm{HL}$, resistance to seeking rehabilitation, and multigenerational households are some factors that may vary across populations and impact these pathways [20]. Tonal language may also play an important but previously underinvestigated role. Understanding Mandarin, for example, depends less on hearing higher frequencies than English and may therefore be less sensitive to the effects of early age-related high-frequency hearing loss [21]. The aims of this study were to assess (i) the association of HL with cognitive impairment among ethnic Chinese Singaporean older adults using visually presented cognitive tests and (ii) how these observed associations compare to those in Western populations.

\section{Methods}

\section{Participants}

The Singapore Longitudinal Ageing Study (SLAS) is a population-based longitudinal study of ageing and health transition of community-dwelling older Singaporeans aged $\geq 55$ years [22]. Around 6,000 participants have been followed up at 3-5 yearly intervals since 2004. SLAS participants who lived close to the study site were invited to participate in the current study.

Peripheral Hearing Loss and Cognition among Chinese Older Adults
Table 1. Sociodemographic characteristics of participants

\begin{tabular}{|c|c|c|}
\hline Variable & \multicolumn{2}{|c|}{ Mean \pm SD or $n(\%)$} \\
\hline Age, years & \multicolumn{2}{|l|}{$71.4 \pm 5.69$} \\
\hline Gender (female) & \multicolumn{2}{|l|}{$155(65.1)$} \\
\hline \multicolumn{3}{|l|}{ Education } \\
\hline Primary & \multicolumn{2}{|l|}{$97(47.1)$} \\
\hline Post-primary & \multicolumn{2}{|l|}{$109(52.9)$} \\
\hline \multicolumn{3}{|l|}{ Hearing levels } \\
\hline Normal hearing & \multicolumn{2}{|l|}{$134(56.8)$} \\
\hline Mild hearing loss & \multicolumn{2}{|l|}{$82(34.7)$} \\
\hline Moderate to severe hearing loss & \multicolumn{2}{|c|}{$\begin{array}{l}20(8.5) \\
\text { (moderate, } n=18 \\
\text { severe, } n=2 \text { ) }\end{array}$} \\
\hline Preferred language & $\begin{array}{l}\text { First } \\
\text { language }\end{array}$ & $\begin{array}{l}\text { Second } \\
\text { language }\end{array}$ \\
\hline Mandarin & $126(52.9)$ & $80(33.6)$ \\
\hline English & $51(21.4)$ & $14(5.9)$ \\
\hline Hokkien & $25(10.5)$ & $77(32.4)$ \\
\hline Cantonese & $23(9.7)$ & $34(14.3)$ \\
\hline Teochew & $8(3.4)$ & $18(7.6)$ \\
\hline Hakka & $4(1.7)$ & $9(3.8)$ \\
\hline Malay & $1(0.4)$ & $2(0.8)$ \\
\hline Hainanese & 0 & $4(1.6)$ \\
\hline
\end{tabular}

Participants were recruited only if they had no known dementia diagnosis and could communicate fluently in English or Mandarin. Subjects who wore hearing aids were excluded as their effect on cognitive performance is unknown.

\section{Measures}

Hearing Assessment

Pure tone audiometry was conducted using a fully automated audiometer (KUDUwave ${ }^{\mathrm{TM}}$; eMoyo, Johannesburg, South Africa) [23]. Air conduction thresholds from 0.25 to $8 \mathrm{kHz}$ were measured. $\mathrm{HL}$ was defined by a BE4PTA (normal $\leq 25 \mathrm{~dB}$; mild 26-40 dB; moderate 41-60 dB; severe 61-80 dB; profound $\geq 81 \mathrm{~dB}$ ) [24]. Moderate HL and severe HL were grouped together due to the small numbers of subjects. A PTA of high frequencies (4 and 8 $\mathrm{kHz}$; BEHPTA) and all frequencies $(0.25-8 \mathrm{kHz}$; BEallPTA) in the better ear was computed to examine the relationship of other measures of peripheral hearing with cognition.

Cognitive Assessment

CANTAB was utilized because it has previously been found to be sensitive to age-related cognitive decline in the local Chinese population, can be presented in languages spoken locally, and is visually presented $[25,26]$. CANTAB was administered using an Apple iPad Air 1. Participants were able to select either English or Mandarin as the test medium. Participants completed the following CANTAB subtests.

Motor Screening Task. It is a brief task to familiarize participants with the touch screen interface. The task tests participants' sensorimotor and comprehension abilities to ensure accurate data 
Table 2. Association of hearing (measured with the better ear 4 frequency PTA $[0.5,1,2$, and $4 \mathrm{kHz}]$ ) and demographic factors with the CANTAB subtest scores

\begin{tabular}{|c|c|c|c|c|c|c|c|c|c|}
\hline \multirow[t]{2}{*}{ Subtest } & \multirow[t]{2}{*}{$N$} & \multicolumn{2}{|l|}{ BE4PTA } & \multicolumn{2}{|l|}{ Age } & \multicolumn{2}{|c|}{ Education } & \multicolumn{2}{|l|}{ Gender } \\
\hline & & $\beta$ & $p$ value & $\beta$ & $p$ value & B & $p$ value & $\beta$ & $p$ value \\
\hline \multicolumn{10}{|l|}{ Memory } \\
\hline PALTEA & 206 & -0.000 & 0.967 & 0.009 & 0.156 & -0.029 & 0.715 & -0.051 & 0.515 \\
\hline PALFAMS & 204 & -0.000 & 0.996 & -0.186 & $0.001^{*}$ & 0.302 & 0.622 & 0.524 & 0.345 \\
\hline VRMFRDS & 183 & -0.002 & 0.449 & -0.016 & $0.014^{*}$ & -0.002 & 0.973 & 0.057 & 0.457 \\
\hline VRMIRTC & 183 & 0.000 & 0.977 & -0.005 & $0.028^{*}$ & 0.013 & 0.484 & 0.002 & 0.936 \\
\hline VRMDRTC & 183 & -0.004 & 0.646 & -0.004 & 0.057 & 0.011 & 0.525 & 0.000 & 0.991 \\
\hline DMSPCS & 205 & -0.005 & 0.753 & -0.011 & 0.744 & -0.164 & 0.646 & -0.445 & 0.219 \\
\hline DMSPCAD & 205 & -0.250 & $0.019^{*}$ & 0.009 & 0.967 & 2.241 & 0.325 & -3.116 & 0.168 \\
\hline SWMBE & 186 & 0.004 & 0.131 & 0.001 & 0.895 & -0.113 & $0.046^{*}$ & 0.087 & 0.184 \\
\hline \multicolumn{10}{|c|}{ Executive function } \\
\hline MTTTIC & 166 & 0.018 & $0.023^{*}$ & 0.032 & $0.041^{*}$ & -0.292 & 0.076 & 0.252 & 0.241 \\
\hline
\end{tabular}

PALTEA, PAL total errors (adjusted); PALFAMS, PAL first attempt memory score; VRMFRDS, VRM free recall; VRMIRTC, VRM immediate recall total correct; VRMDRTC, VRM delayed recall total correct; DMSPCS, DMS percent correct (simultaneous); DMSPCAD, DMS percent correct (all delays); SWMBE, SWM between errors; MTTTIC, MTT total incorrect. * Significance at $p<0.05$.

collection. Participants who were not able to complete the Motor Screening Task due to problems such as visual impairment, inability to comprehend instructions, or dexterity problems were excluded from further participation.

Paired Associates Learning. Paired Associates Learning (PAL) assesses episodic visual memory and learning. Six white boxes change briefly to reveal a pattern which varies in shape and colour. A pattern is then revealed in the centre of the screen. Participants must remember the pattern and match that to a pattern in their memory by touching the box that contains the correct response. The task is made progressively more difficult by presenting 1,2, 3, 6 , and 8 patterns to the participant. The outcome measures are "PAL total errors (adjusted) (PALTEA)" and "PAL first attempt memory score (PALFAMS)." PALTEA is a measure of the total number of errors across all stages, adjusted for each stage not attempted due to previous failure. PALFAMS is the number of correct responses made on the first attempt across all trials.

Verbal Recognition Memory. Verbal Recognition Memory (VRM) assesses immediate and delayed recognition memory and recall. Participants are shown a list of 18 words one at a time and asked to (i) repeat aloud as many as they can immediately after (free recall), (ii) recognize the original words from a list of both new and original words immediately after (immediate), and (iii) recognize the original words from a list of different new and original words after 20-min delay (delayed). The outcome measures for VRM are "VRM free recall: distinct stimuli (VRMFRDS)," "VRM immediate: total correct (VRMIRTC)," and "VRM delayed: total correct (VRMDRTC)."

Multitasking Task. Multitasking Task (MTT) assesses EF and cued attentional set shifting. Participants must touch a box on the left or right of the screen, depending on which side of the screen the arrow appears, or which direction the arrow points at. The words "direction" or "side" will appear on the screen, informing participants which to look out for. The outcome measure is "MTT total incorrect (MTTTIC)."

Delayed Matching to Sample. Delayed Matching to Sample (DMS) tests attention and visual recognition. Participants are shown a complex visual graphic. Four other graphics are then either presented simultaneously with the original graphic or after a delay of 0,4 , or $12 \mathrm{~s}$. Participants must choose the graphic that is identical to the original graphic. The outcome measures are "DMS percent correct (simultaneous) (DMSPCS)" and "DMS percent correct (all delays) (DMSPCAD).”

Spatial Working Memory. Spatial Working Memory (SWM) assesses non-verbal and visuospatial WM and EF. Participants are required to find tokens hidden in either $3,4,6$, or 8 boxes. Participants are informed that there is only 1 token per box and hence should refrain from revisiting a box that was already found to have a token. The outcome measure is "SWM between errors(SWMBE)." SWMBE denotes the number of times a participant revisits a box in which a token has previously been found.

Participants who were not able to understand test instructions for the MTT and SWM subtests were excluded from the MTT and SWM analyses, as the instructions for these tests were more difficult to understand and could directly impact performance. Participants who self-reported reading difficulty were excluded from VRM analyses.

\section{Procedure}

A research assistant was present throughout the assessments for support and further explanation. Participants were randomized to complete the hearing or cognitive assessment first. A Williams Sound Pocketalker (Eden Prairie, MN, USA) personal amplifier was provided for participants with HL, adjusted to comfort level, during the cognition tests and general instructions. 
Table 3. Association of hearing loss (mild, moderate, or greater) and covariates with CANTAB subtest scores

\begin{tabular}{|c|c|c|c|c|c|c|c|c|c|c|}
\hline Subtest & $\beta$ & $p$ value & $\beta$ & $p$ value & $\beta$ & $p$ value & B & $p$ value & $\beta$ & $p$ value \\
\hline PALTEA & 0.004 & 0.958 & -0.223 & 0.124 & 0.011 & 0.091 & -0.041 & 0.612 & -0.069 & 0.352 \\
\hline PALFAMS & -0.454 & 0.474 & 1.779 & 0.088 & -0.200 & $<0.001^{*}$ & 0.291 & 0.633 & 0.632 & 0.250 \\
\hline VRMFRDS & -0.031 & 0.687 & -0.275 & $0.042^{*}$ & -0.014 & $0.038^{*}$ & -0.008 & 0.906 & 0.053 & 0.465 \\
\hline DMSPCS & -0.275 & 0.456 & 0.069 & 0.918 & -0.010 & 0.754 & -0.183 & 0.608 & -0.435 & 0.220 \\
\hline DMSPCAD & -2.700 & 0.242 & -10.600 & 0.019* & -0.005 & 0.980 & 2.424 & 0.272 & -2.677 & 0.230 \\
\hline SWMBE & 0.107 & 0.051 & -0.049 & 0.676 & 0.002 & 0.698 & -0.108 & 0.065 & 0.062 & 0.333 \\
\hline \multicolumn{11}{|l|}{ Executive function } \\
\hline MTTTIC & 0.064 & 0.716 & 0.432 & 0.168 & 0.036 & $0.020 *$ & -0.349 & $0.043^{*}$ & 0.174 & 0.334 \\
\hline
\end{tabular}

$N$ similar to Table 2. Normal hearing is used as the reference category. PAL total errors (adjusted). PALFAMS, PAL first attempt memory score; VRMFRDS, VRM free recall; VRMIRTC, VRM immediate recall total correct; VRMDRTC, VRM delayed recall total correct; DMSPCS, DMS percent correct (simultaneous); DMSPCAD, DMS percent correct (all delays); SWMBE, SWM between errors; MTTTIC, MTT total incorrect. * Significance at $p<0.05$.

\section{Statistical Analysis}

Statistical analyses were performed using STATA version 14.0 [27]. Multiple regression analyses were performed to examine the association between peripheral hearing and cognitive function, adjusted for age, education, and gender. Education was measured categorically as primary of $\leq 6$ years or post-primary of $>6$ years. Peripheral hearing was defined in 3 ways: (1) BE4PTA, (2) BEHPTA, and (3) BEallPTA. All 3 variables were entered as continuous independent variables in separate regression analyses. CANTAB subtest scores were entered as dependent variables. Negative binomial regression was used to model PALTEA, VRMIRTC, MTTTIC, and SWMBE. Poisson regression was used to model VRMFRDS and VRMDRTC. Linear regression was used to model PALFAMS and DMSPCAD. DMSPCS was modelled using logistic regression - due to the distribution of the data, DMSPCS was examined as a binary variable $(100 \%$ correct responses or $<100 \%$ correct responses). Multiple comparisons were not accounted for due to the exploratory nature of the analyses.

\section{Results}

The sociodemographic characteristics of the 238 older adults who participated in the study are shown in Table 1. Two participants did not complete audiometry. All participants spoke at least 1 Chinese tonal language (Table 1).

The significant associations are detailed in Tables $2-5$. In brief, BE4PTA was associated with reduced performance in DMSPCAD $(\beta=-0.25, p=0.019)$ and MTTTIC $(\beta=0.02, p=0.023)$. Moderate to severe HL was associated with reduced performance in DMSPCAD $(\beta=-10.6$, $p=0.019)$ and VRMFRDS $(\beta=-0.28, p=0.042)$.
BEHPTA was associated with reduced performance in the SWMBE $(\beta=0.004, p=0.022)$. All-frequency HL was associated with reduced performance in spatial WM and multitasking $(\beta=0.01, p=0.040$, and $\beta=0.02, p=0.048)$.

\section{Discussion}

Our results contribute domain-specific data obtained using a non-verbally administered cognitive test battery in an ethnic Chinese population to the literature on the association between HL and cognitive function. New data are presented pertaining to how HL in different frequency ranges affects cognition in a tonal language-speaking population.

Hearing was a predictor of performance on VRM, SWM, MTT, and DMS tests that required verbal, nonverbal, and visuospatial WM, attention, and EF after adjusting for age, education, and gender. WM, EF, and attention are all important for speech comprehension and deteriorate with age $[15,28]$. WM becomes increasingly important in difficult listening situations in older people, even when their peripheral hearing is normal [29]. Yet further demand is placed on WM, EF, and attention to aid speech perception in older people with HL, resulting in poorer performance in tests of these domains as resources become overwhelmed. It is notable that altered EF is found in early Alzheimer's disease [30].

Interethnic differences in dementia and HL have previously been identified, and this relationship therefore 
Table 4. Association of hearing (measured with the better ear high-frequency PTA [ 4 and $8 \mathrm{kHz}]$ ) and demographic factors with CANTAB subtest scores

\begin{tabular}{|c|c|c|c|c|c|c|c|c|}
\hline Subtest & \multicolumn{2}{|l|}{ BEHPTA } & \multicolumn{2}{|l|}{ Age } & \multicolumn{2}{|c|}{ Education } & \multicolumn{2}{|l|}{ Gender } \\
\hline PALTEA & 0.002 & 0.452 & 0.007 & 0.288 & -0.018 & 0.825 & -0.026 & 0.744 \\
\hline PALFAMS & -0.010 & 0.584 & -0.173 & $0.003^{*}$ & 0.261 & 0.664 & 0.409 & 0.471 \\
\hline VRMFRDS & -0.000 & 0.921 & -0.017 & $0.005^{*}$ & 0.006 & 0.926 & 0.068 & 0.391 \\
\hline DMSPCS & 0.002 & 0.837 & 0.037 & $0.021^{*}$ & -0.132 & 0.709 & -0.388 & 0.292 \\
\hline DMSPCAD & -0.082 & 0.238 & -0.017 & 0.601 & 2.855 & 0.205 & -2.564 & 0.273 \\
\hline SWMBE & 0.004 & $0.022 *$ & -0.081 & 0.686 & -0.109 & 0.059 & 0.103 & 0.124 \\
\hline \multicolumn{9}{|l|}{ Executive function } \\
\hline MTTाC & 0.005 & 0.395 & -0.007 & $0.009 *$ & -0.341 & $0.045^{*}$ & 0.172 & 0.369 \\
\hline
\end{tabular}

N similar to Table 2. PAL total errors (adjusted). PALFAMS, PAL first attempt memory score; VRMFRDS, VRM free recall; VRMIRTC, VRM immediate recall total correct; VRMDRTC, VRM delayed recall total correct; DMSPCS, DMS percent correct (simultaneous); DMSPCAD, DMS percent correct (all delays); SWMBE, SWM between errors; MTTTIC, MTT total incorrect. * Significance at $p<0.05$.

Table 5. Association of hearing (measured with the better ear all-frequency PTA [0.25-8 kHz]) and demographic factors with CANTAB subtest scores

\begin{tabular}{|c|c|c|c|c|c|c|c|c|}
\hline & $\beta$ & $p$ value & $\beta$ & $p$ value & $\beta$ & $p$ value & $\beta$ & $p$ value \\
\hline PALTEA & 0.003 & 0.541 & 0.007 & 0.271 & -0.017 & 0.833 & -0.031 & 0.692 \\
\hline PALFAMS & -0.011 & 0.722 & -0.177 & $0.002^{*}$ & 0.265 & 0.664 & 0.450 & 0.425 \\
\hline VRMFRDS & -0.003 & 0.350 & -0.015 & 0.019* & -0.004 & 0.954 & 0.051 & 0.513 \\
\hline DMSPCS & 0.000 & 0.987 & -0.014 & 0.667 & -0.144 & 0.686 & -0.415 & 0.255 \\
\hline DMSPCAD & -0.190 & 0.077 & -0.023 & 0.909 & 2.531 & 0.268 & -2.892 & 0.214 \\
\hline SWMBE & 0.006 & $0.040^{*}$ & -0.001 & 0.883 & -0.109 & 0.055 & 0.100 & 0.133 \\
\hline \multicolumn{9}{|c|}{ Executive function } \\
\hline MTTTIC & 0.017 & $0.048^{*}$ & 0.031 & $0.047^{*}$ & -0.301 & 0.062 & 0.244 & 0.276 \\
\hline
\end{tabular}

N similar to Table 2. PAL total errors (adjusted). PALFAMS, PAL first attempt memory score; VRMFRDS, VRM free recall; VRMIRTC, VRM immediate recall total correct; VRMDRTC, VRM delayed recall total correct; DMSPCS, DMS percent correct (simultaneous); DMSPCAD, DMS percent correct (all delays); SWMBE, SWM between errors; MTTIC, MTT total incorrect. * Significance at $p<0.05$.

warrants comprehensive study [31, 32]. Most of the published literature from Asia has used cognitive screening tools or mild cognitive impairment/dementia diagnosis, which provides little domain-specific information [17, 18, 33-35]. Furthermore, most Asian studies on cognitive impairment and HL are based in part on verbally loaded cognition tests. Deal et al. [3] found that the exclusion of cognitive tests that solely utilized auditory stimuli from domain summary scores resulted in a significant differ- ence in memory performance. Our findings largely mirror the limited domain-specific data that are available in ethnic Chinese subjects. Ren et al. [36] found peripheral hearing to be associated with tests of EF, verbal learning and memory, psychomotor speed, and attention in a Han Chinese population. Our results are also similar to research done on Australian, European, and American participants $[9,15,37]$. Direct comparison can be made with an English-speaking Australian population, in whom 
similar CANTAB tests were utilized [16]. Spatial WM was also found to be significantly associated with worse HL. However, attention and non-verbal recall and recognition tasks were not affected. This may reflect the different frequency ranges that were used for analysis or represent real differences in the effect of HL on the two populations.

Differences in the acoustic properties of language could potentially impact this relationship. Variation in pitch serves to convey lexical meaning in tonal languages [38]. Many Mandarin speech sounds are clustered together in frequency and intensity between 0.5 and $2 \mathrm{kHz}$ so may be easily confused in patients with HL; conversely, Mandarin speakers may be less affected by early age-related high-frequency hearing loss [21]. Our results suggest that in this mixed tonal language-speaking population, WM is the first cognitive domain to become overwhelmed, as evidenced by poorer performance on tests of WM in subjects with high-frequency HL. Further demand is placed on cognitive processes utilized for speech processing such as attention and EF when HL progresses to involve the middle and low frequencies, resulting in increasing difficulty discriminating Mandarin tones [21].

Education has been consistently shown to predict cognitive performance and dementia risk [39, 40]. Less education is also associated with worse performance in CAN$\mathrm{TAB}$ tests of visual sustained attention, reaction time, and learning ability [41]. However, education did not predict performance on most CANTAB subtests in our study. Our results also indicate that age was a more consistent predictor of cognitive performance than hearing in this population. Age has previously been reported as a stronger predictor than hearing of some domains such as processing speed and inhibition [15]. In the present study, age could have been a more consistent predictor because participants needed to use an iPad. Nevertheless, the associations of HL with cognitive parameters reported are comparable to other risk factors for cognitive decline and dementia in the existing literature [5].

Our study has several limitations. Instructions for the CANTAB tests were presented in Taiwanese Mandarin, which is slightly different to the Standard Mandarin used locally in Singapore. This might have contributed to difficulty in understanding certain test instructions, thus reducing the power of some analyses. Second, the sample of all hearing-impaired individuals was small and precluded subgroup analyses on participants with moderate to severe HL. Third, the study subjects, despite being Chinese, were heterogeneous in that they spoke a mixture of several different Chinese dialects, all of which may have different acoustic properties. Finally, the study is cross-sectional in nature and provides only a static snapshot of Singaporean older adults. Objective hearing assessments and visually presented cognitive assessments should be administered longitudinally. Studies comparing homogeneous populations of tonal and non-tonal language speakers would also be of interest.

\section{Conclusion}

The present study is one of the few studies globally which uses non-hearing-dependent cognitive assessments to estimate the relationship between peripheral hearing and cognitive functioning among older adults. HL was associated with worse performance in tasks requiring WM and EF in this tonal language-speaking population, as in many Western populations.

\section{Statement of Ethics}

The study was approved by the National Healthcare Group Domain Specific Review Board (NHG DSRB Ref: 2016/00962). All participants gave written informed consent.

\section{Conflict of Interest Statement}

$\mathrm{Ng}$ Tze Pin is an Editorial Board member for Dementia and Geriatric Cognitive Disorders. The other authors have no conflicts of interest to declare.

\section{Funding Sources}

The study was supported by research grant funding from JurongHealth Research and Development Fund (16-49). The fund holders had no role in the preparation of the data or manuscript.

\section{Author Contributions}

S.L.W., T.P.N., and R.L.H. conceptualized and supervised the research, reviewed and edited the final $\mathrm{draft}$, and acquired funding for the research. S.O.N. and E.J.K. curated data, analysed data, managed the project, prepared the original draft, and reviewed and edited the final draft. F.L., R.E., and D.J. provided external mentorship to the core team, contributed to study design and data interpretation, and reviewed and edited the final draft.

\section{Data Availability Statement}

The data that support the findings of this study are available upon written request to the corresponding author. 


\section{References}

1 Valentijn SA, van Boxtel MP, van Hooren SA, Bosma H, Beckers HJ, Ponds RW, et al. Change in sensory functioning predicts change in cognitive functioning: results from a 6-year followup in the Maastricht aging study. J Am Geriatr Soc. 2005 Mar;53(3):374-80.

2 Lin FR. Hearing loss and cognition among older adults in the United States. J Gerontol A Biol Sci Med Sci. 2011 Oct;66(10):1131-6

3 Deal JA, Sharrett AR, Albert MS, Coresh J, Mosley TH, Knopman D, et al. Hearing impairment and cognitive decline: a pilot study conducted within the atherosclerosis risk in communities neurocognitive study. Am J Epidemiol. 2015 May 1;181(9):680-90.

4 Taljaard DS, Olaithe M, Brennan-Jones CG, Eikelboom RH, Bucks RS. The relationship between hearing impairment and cognitive function: a meta-analysis in adults. Clin Otolaryngol. 2016 Dec;41(6):718-29.

5 Loughrey DG, Kelly ME, Kelley GA, Brennan S, Lawlor BA. Association of age-related hearing loss with cognitive function, cognitive impairment, and dementia: a systematic review and meta-analysis. JAMA Otolaryngol Head Neck Surg. 2018 Feb 1;144(2):115-26.

6 Lin FR, Metter EJ, O’Brien RJ, Resnick SM, Zonderman AB, Ferrucci L. Hearing loss and incident dementia. Arch Neurol. 2011 Feb; 68(2):214-20.

7 Deal JA, Betz J, Yaffe K, Harris T, PurchaseHelzner E, Satterfield S, et al. Hearing impairment and incident dementia and cognitive decline in older adults: the health ABC study. J Gerontol A Biol Sci Med Sci. 2017 May 1; 72(5):703-9.

8 Wayne RV, Johnsrude IS. A review of causal mechanisms underlying the link between agerelated hearing loss and cognitive decline. Ageing Res Rev. 2015 Sep;23(Pt B):154-66.

9 Nixon G, Sarant JZ, Tomlin D, Dowell R. The relationship between peripheral hearing loss and higher order listening function on cognition in older Australians. Int J Audiol. 2019 Dec;58(12):933-44.

10 Wong LL, Yu JK, Chan SS, Tong MC. Screening of cognitive function and hearing impairment in older adults: a preliminary study. Biomed Res Int. 2014;2014:867852.

11 Jorgensen LE, Palmer CV, Pratt S, Erickson KI, Moncrieff D. The effect of decreased audibility on MMSE performance: a measure commonly used for diagnosing dementia. J Am Acad Audiol. 2016 Apr;27(4):311-23.

12 Uchida Y, Sugiura S, Nishita Y, Saji N, Sone M, Ueda H. Age-related hearing loss and cognitive decline: the potential mechanisms linking the two. Auris Nasus Larynx. 2019 Feb;46(1):1-9.

13 Lin VY, Chung J, Callahan BL, Smith L, Gritters N, Chen JM, et al. Development of cognitive screening test for the severely hearing impaired: hearing-impaired MoCA. Laryngoscope. 2017 May;127(Suppl 1):S4-S11.

14 Claes AJ, Van de Heyning P, Gilles A, HofkensVan den Brandt A, Van Rompaey V, Mertens
G. Impaired cognitive functioning in cochlear implant recipients over the age of 55 years: a cross-sectional study using the repeatable battery for the assessment of neuropsychological status for hearing-impaired individuals (RBANS-H). Front Neurosci. 2018;12:580.

15 Volter C, Gotze L, Falkenstein M, Dazert S, Thomas JP. Application of a computer-based neurocognitive assessment battery in the elderly with and without hearing loss. Clin Interv Aging. 2017;12:1681-90.

16 Jayakody DMP, Friedland PL, Eikelboom RH, Martins RN, Sohrabi HR. A novel study on association between untreated hearing loss and cognitive functions of older adults: baseline non-verbal cognitive assessment results. Clin Otolaryngol. 2018 Feb;43(1):182-91.

17 Luo Y, He P, Guo C, Chen G, Li N, Zheng X. Association between sensory impairment and dementia in older adults: evidence from China. J Am Geriatr Soc. 2018 Mar;66(3):480-6.

18 Gyanwali B, Hilal S, Venketasubramanian N, Chen C, Loo JHY. Hearing handicap in Asian patients with dementia. Am J Otolaryngol. 2020 Mar;41(2):102377.

19 Lin FR. Making sense of the senses in aging research. J Gerontol A Biol Sci Med Sci. 2020 Feb 14;75(3):529-30.

20 Roystonn K, Abdin E, Shahwan S, Zhang Y, Sambasivam R, Vaingankar JA, et al. Living arrangements and cognitive abilities of community-dwelling older adults in Singapore. Psychogeriatrics. 2020 Sep;20(5):625-35.

$21 \mathrm{Hu}$ XJ, Li FF, Lau CC. Development of the Mandarin speech banana. Int J Speech Lang Pathol. 2019 Aug;21(4):404-11.

22 Feng L, Ng TP, Chuah L, Niti M, Kua EH. Homocysteine, folate, and vitamin B-12 and cognitive performance in older Chinese adults: findings from the Singapore longitudinal ageing study. Am J Clin Nutr. 2006 Dec;84(6):1506-12.

23 Swanepoel de W, Mngemane S, Molemong S, Mkwanazi $\mathrm{H}$, Tutshini S. Hearing assessment-reliability, accuracy, and efficiency of automated audiometry. Telemed J E Health. 2010 Jun;16(5):557-63.

24 World Health Organization. Report of the informal working group on prevention of deafness and hearing impairment programme planning. Geneva: World Health Organization; 1991. Available from: https://apps.who. int/iris/handle/10665/58839?show=full.

25 Cambridge Cognition Limited. CANTABeclipse test administration guide. Cambridge: Cambridge Cognition Limited; 2012.

26 Lee A, Archer J, Wong CK, Chen SH, Qiu A. Age-related decline in associative learning in healthy Chinese adults. PLoS One. 2013; 8(11):e80648.

27 StataCorp. Stata statistical software: release 14 (computer program); 2015.

28 Jayakody DMP, Friedland PL, Martins RN, Sohrabi HR. Impact of aging on the auditory system and related cognitive functions: a narrative review. Front Neurosci. 2018;12:125.
29 Meister H, Schreitmüller S, Grugel L, Ortmann $\mathrm{M}$, Beutner D, Walger M, et al. Cognitive resources related to speech recognition with a competing talker in young and older listeners. Neuroscience. 2013 Mar 1;232:74-82.

30 Nathan J, Wilkinson D, Stammers S, Low JL. The role of tests of frontal executive function in the detection of mild dementia. Int J Geriatr Psychiatry. 2001 Jan;16(1):18-26.

31 Agrawal Y, Platz EA, Niparko JK. Prevalence of hearing loss and differences by demographic characteristics among US adults: data from the national health and nutrition examination survey, 1999-2004. Arch Intern Med. 2008 Jul 28;168(14):1522-30.

32 Venketasubramanian N, Sahadevan S, Kua $\mathrm{EH}$, Chen $\mathrm{CP}, \mathrm{Ng} \mathrm{TP}$. Interethnic differences in dementia epidemiology: global and AsiaPacific perspectives. Dement Geriatr Cogn Disord. 2010;30(6):492-8.

33 Sugawara N, Sasaki A, Yasui-Furukori N, Kakehata S, Umeda T, Namba A, et al. Hearing impairment and cognitive function among a community-dwelling population in Japan. Ann Gen Psychiatry. 2011 Oct 1;10(1):27.

34 Heywood R, Gao Q, Nyunt MSZ, Feng L, Chong MS, Lim WS, et al. Hearing loss and risk of mild cognitive impairment and dementia: findings from the Singapore longitudinal ageing study. Dement Geriatr Cogn Disord. 2017;43(5-6):259-68.

35 Lim MYL, Loo JHY. Screening an elderly hearing impaired population for mild cognitive impairment using mini-mental state examination (MMSE) and Montreal cognitive assessment (MoCA). Int J Geriatr Psychiatry. 2018 Jul;33(7):972-9.

36 Ren F, Luo J, Ma W, Xin Q, Xu L, Fan Z, et al. Hearing loss and cognition among older adults in a Han Chinese cohort. Front Neurosci. 2019;13:632.

37 Harrison Bush AL, Lister JJ, Lin FR, Betz J, Edwards JD. Peripheral hearing and cognition: evidence from the staying keen in later life (SKILL) study. Ear Hear. 2015 Jul;36(4): 395-407.

38 Ge J, Peng G, Lyu B, Wang Y, Zhuo Y, Niu Z, et al. Cross-language differences in the brain network subserving intelligible speech. Proc Natl Acad Sci USA. 2015 Mar 10;112(10): 2972-7.

39 Caamano-Isorna F, Corral M, Montes-Martinez A, Takkouche B. Education and dementia: a meta-analytic study. Neuroepidemiology. 2006;26(4):226-32.

40 Meng X, D'Arcy C. Education and dementia in the context of the cognitive reserve hypothesis: a systematic review with meta-analyses and qualitative analyses. PLoS One. 2012; 7(6):e38268.

41 Bento-Torres NV, Bento-Torres J, Tomás $\mathrm{AM}$, Costa VO, Corrêa PG, Costa CN, et al. Influence of schooling and age on cognitive performance in healthy older adults. Braz J Med Biol Res. 2017 Mar 23;50(4):e5892. 